\title{
Massively Parallel Sequencing of a Chinese Family with DFNA9 Identified a Novel Missense Mutation in the LCCL Domain of COCH
}

\author{
Xiaodong Gu, ${ }^{1,2}$ Wenling Su, ${ }^{3}$ Mingliang Tang, ${ }^{4,5}$ Luo Guo, ${ }^{1,2,6}$ \\ Liping Zhao, ${ }^{1,2,6}$ and Huawei $\mathrm{Li}^{1,2,7,8,9}$ \\ ${ }^{1}$ Otorhinolaryngology Department, Affiliated Eye and ENT Hospital, State Key Laboratory of Medical Neurobiology, \\ Fudan University, Shanghai 200031, China \\ ${ }^{2}$ Key Laboratory of Hearing Medicine of National Health and Family Planning Commission, Shanghai 200031, China \\ ${ }^{3}$ Department of Otolaryngology-Head and Neck Surgery, The First Affiliated Hospital, Xiamen University, Xiamen, China \\ ${ }^{4}$ Key Laboratory for Developmental Genes and Human Disease, Ministry of Education, Institute of Life Sciences, \\ Southeast University, Nanjing 210096, China \\ ${ }^{5}$ Co-Innovation Center of Neuroregeneration, Nantong University, Nantong 226001, China \\ ${ }^{6}$ Central Laboratory, Affiliated Eye and ENT Hospital, Fudan University, Shanghai 200031, China \\ ${ }^{7}$ Institutes of Biomedical Sciences, Fudan University, Shanghai 200032, China \\ ${ }^{8}$ The Institutes of Brain Science and the Collaborative Innovation Center for Brain Science, Fudan University, Shanghai, China \\ ${ }^{9}$ Shanghai Engineering Research Center of Cochlear Implant, Shanghai 200031, China
}

Correspondence should be addressed to Huawei Li; hwli@shmu.edu.cn

Received 9 September 2016; Revised 16 November 2016; Accepted 23 November 2016

Academic Editor: Jian Wang

Copyright (C) 2016 Xiaodong Gu et al. This is an open access article distributed under the Creative Commons Attribution License, which permits unrestricted use, distribution, and reproduction in any medium, provided the original work is properly cited.

DFNA9 is a late-onset, progressive, autosomal dominantly inherited sensorineural hearing loss with vestibular dysfunction, which is caused by mutations in the $\mathrm{COCH}$ (coagulation factor $\mathrm{C}$ homology) gene. In this study, we investigated a Chinese family segregating autosomal dominant nonsyndromic sensorineural hearing loss. We identified a missense mutation c.T275A p.V92D in the LCCL domain of $\mathrm{COCH}$ cosegregating with the disease and absent in 100 normal hearing controls. This mutation leads to substitution of the hydrophobic valine to an acidic amino acid aspartic acid. Our data enriched the mutation spectrum of DFNA9 and implied the importance for mutation screening of $\mathrm{COCH}$ in age related hearing loss with vestibular dysfunctions.

\section{Introduction}

As the most common sensory impairment, hearing loss (HL) affects one of every 500 newborn infants, and its prevalence rises to 2.7 per 1000 children before the age of 5 and 3.5 per 1000 during adolescence $[1,2]$. HL is a genetically and clinically heterozygous disorder and can be classified according to pattern of inheritance (autosomal dominant, autosomal recessive, or X-linked recessive, and mitochondrial inheritance), the absence (nonsyndromic) or presence (syndromic) of other clinical features, and age at onset (prelingual or postlingual) [3]. To date, researchers have identified 98 genes associated with nonsyndromic hearing loss (NSHL) (Hereditary Hearing Loss Homepage, http://hereditaryhearingloss .org). Most of the mutations in the autosomal dominant loci cause postlingual hearing impairments [4].

Mutations in the $\mathrm{COCH}$ gene are responsible for the lateonset, progressive ADNSHL with incomplete penetrance of vestibular malfunction known as DFNA9 [5]. The $\mathrm{COCH}$ gene encodes a 550 -aa extracellular protein cochlin that is the most highly expressed protein in the human and mouse inner ear [6]. The cochlin amino acid sequence contains an $\mathrm{N}$ terminal signal peptide, an LCCL domain highly homologous to factor $\mathrm{C}$, a serine proteinase involved in immune response 


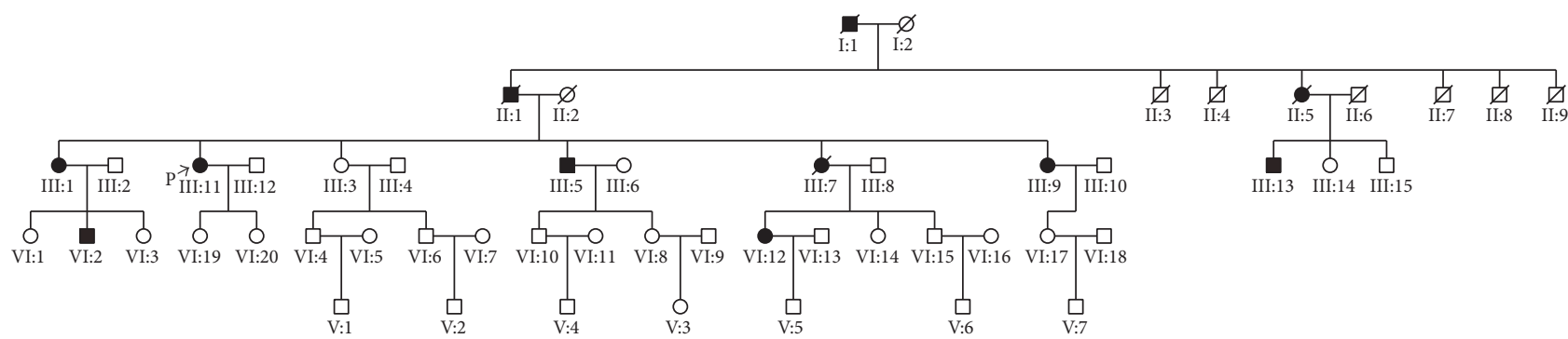

(a)
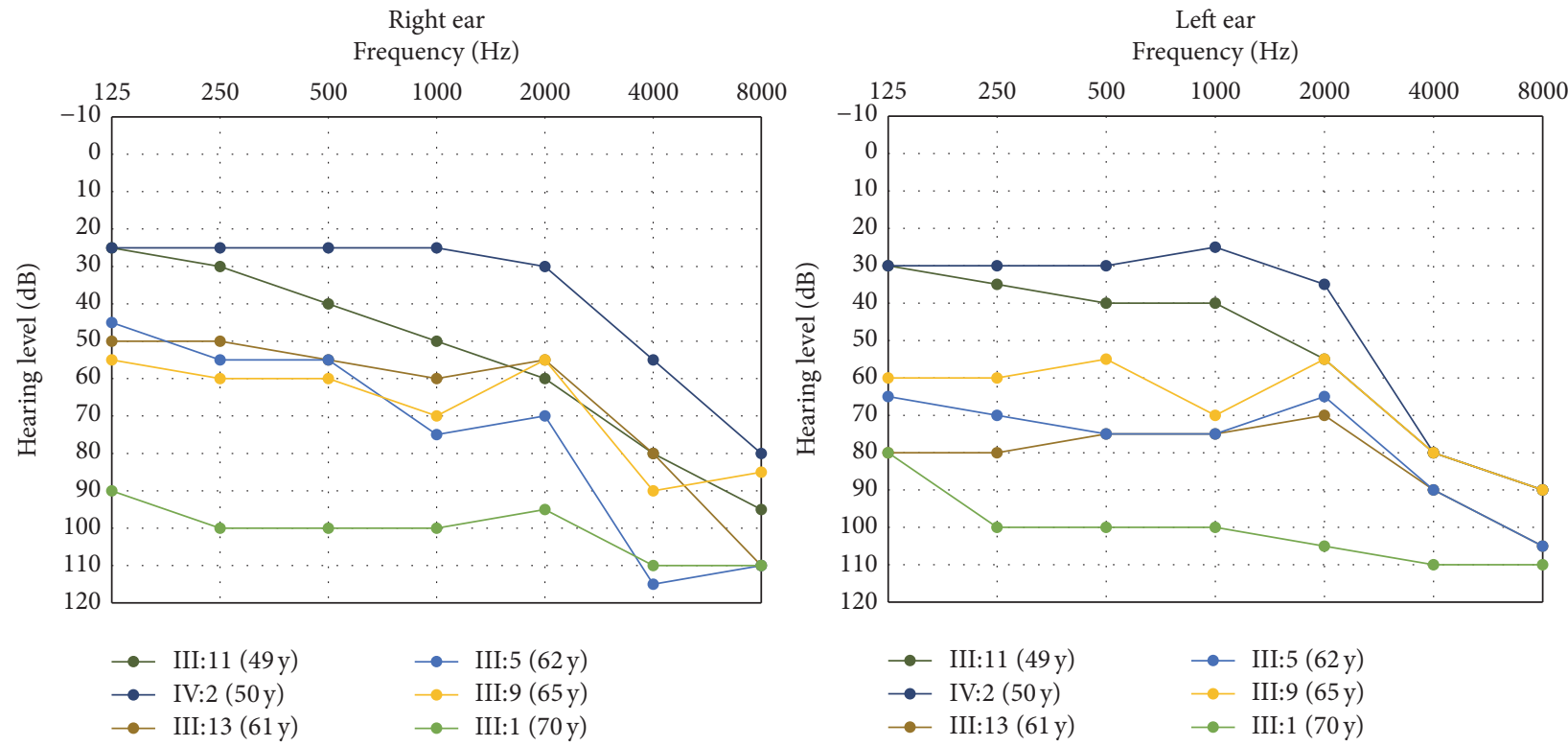

(b)

FIGURE 1: (a) Pedigree of the family with nonsyndromic autosomal recessive hearing loss. Darkened symbols denote affected individuals. (b) Audiograms of all affected individuals in the family.

of the Limulus, and two von Willebrand factor A (vWFA) domains [7]. So far, twenty-three DFNA9 mutations were identified in the LCCL domain, vWFA domain, and intervening domain [8-15]. Individuals with DFNA9 manifest lateonset progressive HL with onset usually before the fourth or fifth decade [16]. Onset of HL is initially in high frequencies, progressing to include lower frequencies, and usually leads to severe HL by the sixth decade of life. $\mathrm{COCH}$ mutations also result in vestibular dysfunction and balance problems in many affected individuals [16].

In this study, we performed massively parallel sequencing on a nonconsanguineous Chinese family segregating autosomal dominant sensorineural HL and identified a novel missense mutation in the LCCL domain.

\section{Results}

2.1. Clinical Phenotype. Seven individuals in family G405 were diagnosed with sensorineural hearing loss by otologic and audiometric analysis (Figure 1(a)). Affected family members showed moderate to severe bilateral sensorineural hearing loss initially affected high frequencies and, with increasing age, developed to mid- and low-frequencies, which resulted in a flat or downward sloping audiogram (Figure 1(b)). The self-reported age at onset of hearing loss was in the 2 nd or 3rd decade. Vertigo, dizziness, and tinnitus have been reported or diagnosed in all affected family members except for VI:12.

2.2. Massively Parallel Sequencing. Targeted MPS aiming 131 deafness-associated genes was applied to proband of family G123. A mean depth of coverage of 176 was achieved with more than $98.6 \%$ of the targeted bases covered by more than 10 reads. Variants were called and filtered using strategies described previously. Briefly, variants meeting the following criteria were filtered out: (1) variants covered by less than 10 sequencing reads; (2) intronic and synonymous variants; (3) variants with allele frequencies bellow 0.01 in 1000 genome database, ESP6500 database, or ExAC database. Consequently, the COCH mutation c.T275A p.V92D (NM_001135058) was the only candidate variant that passed all filtering steps. 


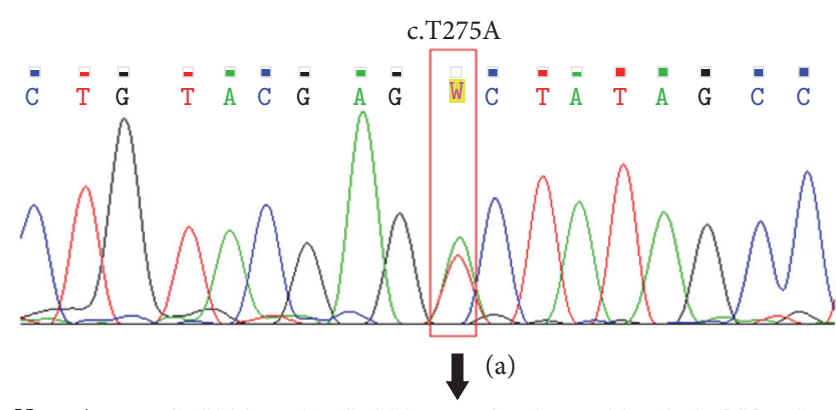

H. sapiens SICGAAVHRGVISNSGGPVRVYSLPGRENYSSVDANGIQSQMLSRW P. troglodytes SICGAAVHRGVISNSGGPVRVYSLPGRENYSSVDANGIQSQMLSRW M. mulatta SICGAAVHRGVISNSGGPVRVYSLPGRENYSSVDANGIQSQMLSRW M. musculus SICGAAVHRGVIGTSGGPVRVYSLPGRENYSSVDANGIQSQMLSRW G. gallus SVCGAAIHRGVITNAGGAVRVQTLPGQENYPAVHANGIQSQVLSRW D. rerio SICGAAIHRGIIGLSGGPVEVHGLQGRTNYLSSYAHGVQSQSLSQW (b)

\begin{tabular}{lcccc}
\hline & SIFT & PolyPhen2 & LRT & MutationTaster \\
\hline p.V92D & 0(D) & 0.903(D) & 0.999998(D) & 0.999978(D) \\
\hline
\end{tabular}

(c)

Figure 2: (a) Sanger sequencing chromatograms showing the c.T275A p.V92D mutation of the family. (b) Protein sequence alignment showing conservation of the V92 residue in cochlin across human (H. sapiens), chimpanzee (P. troglodytes), macaca ( $M$. mulatta), mouse (M. musculus), chicken (G. gallus), and zebrafish (D. rerio). (c) Pathogenicity prediction using computational programs. $\mathrm{D}$ in the parentheses stands for deleterious.

2.3. Mutation Analysis. Segregation analysis of candidate variants was carried out by Sanger sequencing. The novel COCH mutation c.T275A:p.V92D (NM_001135058) (Figure 2(a)) was present in all affected individuals but not in the normal family members that are above 40 years of age. This mutation caused an amino acid substitution in the LCCL domain of cochlin protein. Sequence alignment analysis of $\mathrm{COCH}$ from different species revealed that this amino acid is highly conserved (Figure 2(b)). The mutation was predicted to be deleterious by four computational programs (Figure 2(c)). Then, we scanned exon 4 of $\mathrm{COCH}$ gene in 100 unrelated Chinese control individuals. Consequently, no variants were detected.

\section{Methods}

3.1. Subjects and Clinical Diagnosis. A Chinese nonconsanguineous family, G405, with late-onset progressive hearing loss was recruited from the Department of Otolaryngology, Affiliated Eye and ENT Hospital, Fudan University, Shanghai, China. Family G405 has five generations and segregated late-onset autosomal dominant sensorineural hearing loss by otologic and audiometric analysis. Twenty-five family members, including seven affected individuals, were recruited. Otoscopy and pure tone audiometry (including frequencies from 250 to $8000 \mathrm{~Hz}$ ) were applied to identify the phenotype. Vestibular function was assessed in some family members by caloric testing and electronystagmography. Blood samples were drawn from the participants. Written informed consent was obtained from all participating individuals in accordance with the ethics committee of Fudan University.
3.2. Massively Parallel Sequencing. Genomic DNA was extracted from whole blood using genomic DNA isolation kit (Qiagen, Hilden, Germany). Exome capture was carried out using TruSeq Exome Enrichment Kit according to the manufacturer's protocols. Captured libraries were then loaded onto the Hiseq2000 platform, and sequencing was performed $100 \mathrm{bp}$ paired-end, providing at least 80 -fold coverage for each sample. Raw image files were processed by Illumina's Cassava pipeline for base-calling with default parameters. The proband of family G123 was subjected to a gene panel containing 131 deafness genes. Capture and MPS of the coding exons plus $\sim 100$ bp of the flanking intronic sequences for the 131 deafness genes on a HiSeq2000 (Illumina) were performed by Otogenetics Corporation (Norcross, GA). A total of $3 \mu \mathrm{g}$ genomic DNA was used as input material for NimbleGen capture methods to generate $2 \times 100$ paired-end reads. Sequencing reads were aligned to human reference genome (hg19/NCBI 37) using the Burrows-Wheeler Aligner (BWA) program, refined using the Genome Analysis Tool Kit (GATK) software and Picard. The genotypes in target regions were identified using the GATK Unified Genotyper; quality scores of the variants were recalibrated. Functional annotations of the variants were performed by Annovar [17].

3.3. Mutational Analysis. Segregation analysis of the candidate mutation identified by MPS was performed in available members of family G405 using PCR followed by bidirectional Sanger sequencing of the amplified fragments (ABI 3730XL; Applied Biosystems, Foster City, CA). In addition, sequences from 100 ethnicity-matched samples with normal hearing were examined.

\section{Discussion}

In this study, we identified a novel heterozygous missense mutation within the LCCL domain of cochlin in a large Chinese family with late-onset progressive sensorineural $\mathrm{HL}$ and vestibular dysfunction. The mutations cosegregated with the disease and were not observed in public databases or 100 ethnicity-matched controls.

The p.V92D mutation identified in this study resides within the N-terminal LCCL domain of cochlin, which leads to substitution of the hydrophobic valine to an acidic amino acid aspartic acid. Mutations of this domain have been shown to cause misfolding and aggregation of the cochlin protein in a dominant-negative fashion and lead to cytotoxicity $[7,16]$. It is noteworthy that most of the patients carrying mutations in the LCCL domain manifested vestibular dysfunctions. Our study supported this genotype-phenotype correlation because nearly all affected patients of family G405 exhibit vestibular dysfunction.

The pathogenic mechanisms of DFNA9 mutations have not been fully revealed; recent studies suggested several possible mechanisms. Several studies suggest that mutations in the LCCL domain induce misfolded LCCL domain and demonstrate cytotoxicity leading to inner ear damage $[6,18]$. Others demonstrated that mutant cochlin forms a stable dimer that is sensitive to reducing agent. The mutant cochlin can stabilize wild type cochlin in the dimer conformation, 
providing a possible explanation for the dominant nature of DFNA9 mutations [19]. A recent study indicates that amino acid substitutions in cochlin lessened cochlin susceptibility to cleavage enzyme induced by aggrecanase, which caused reduced secretion of the LCCL domain to the extracellular compartment [8]. It is possible that the p.V92D mutation in our study may also lead to cochlin misfolding.

In conclusion, our findings enriched the mutation and genotype-phenotype correlation spectrum of DFNA9 and implied the importance for mutation screening of $\mathrm{COCH}$ in late-onset hearing loss with vestibular dysfunctions.

\section{Competing Interests}

The authors declare that they have no competing interests.

\section{Authors' Contributions}

Xiaodong Gu, Wenling Su, Mingliang Tang, and Luo Guo contributed equally to this work.

\section{Acknowledgments}

This work was supported by grants from the Major State Basic Research Development Program of China (2016YFC0905200 and 973 Program Grant 2015CB965000), the National Natural Science Foundation of China (Grants 81470687, 81230019, and 81500801), the Construction Program of the Shanghai Committee of Science and Technology (Grant 12DZ2251700), and the Xiamen Science and Technology Planning Project (Grant 3502z2014013).

\section{References}

[1] C. C. Morton and W. E. Nance, "Newborn hearing screeninga silent revolution," The New England Journal of Medicine, vol. 354, no. 20, pp. 2151-2164, 2006.

[2] N. G. Robertson, A. B. Skvorak, Y. Yin et al., "Mapping and characterization of a novel cochlear gene in human and in mouse: a positional candidate gene for a deafness disorder, DFNA9," Genomics, vol. 46, no. 3, pp. 345-354, 1997.

[3] A. E. Shearer and R. J. Smith, "Genetics: advances in genetic testing for deafness," Current Opinion in Pediatrics, vol. 24, no. 6, pp. 679-686, 2012.

[4] N. Hilgert, R. Smith, and G. Camp, "Function and expression pattern of nonsyndromic deafness genes," Current Molecular Medicine, vol. 9, no. 5, pp. 546-564, 2009.

[5] N. G. Robertson, L. Lu, S. Heller et al., "Mutations in a novel cochlear gene cause DFNA9, a human nonsyndromic deafness with vestibular dysfunction," Nature Genetics, vol. 20, no. 3, pp. 299-303, 1998.

[6] T. Ikezono, A. Omori, S. Ichinose, R. Pawankar, A. Watanabe, and T. Yagi, "Identification of the protein product of the Coch gene (hereditary deafness gene) as the major component of bovine inner ear protein," Biochimica et Biophysica Acta (BBA)-Molecular Basis of Disease, vol. 1535, no. 3, pp. 258-265, 2001.

[7] N. G. Robertson, C. W. R. J. Cremers, P. L. M. Huygen et al., "Cochlin immunostaining of inner ear pathologic deposits and proteomic analysis in DFNA9 deafness and vestibular dysfunction," Human Molecular Genetics, vol. 15, no. 7, pp. 10711085, 2006.

[8] J. Jung, H. S. Kim, M. G. Lee, E. J. Yang, and J. Y. Choi, "Novel COCH p.V123E mutation, causative of DFNA9 sensorineural hearing loss and vestibular disorder, shows impaired cochlin post-translational cleavage and secretion," Human Mutation, vol. 36, no. 12, pp. 1168-1175, 2015.

[9] D.-Y. Chen, Y.-C. Chai, T. Yang, and H. Wu, "Clinical characterization of a novel COCH mutation G87V in a Chinese DFNA9 family," International Journal of Pediatric Otorhinolaryngology, vol. 77, no. 10, pp. 1711-1715, 2013.

[10] T. Makishima, C. I. Rodriguez, N. G. Robertson, C. C. Morton, C. L. Stewart, and A. J. Griffith, "Targeted disruption of mouse Coch provides functional evidence that DFNA9 hearing loss is not a COCH haploinsufficiency disorder," Human Genetics, vol. 118, no. 1, pp. 29-34, 2005.

[11] I. Nagy, M. Horváth, M. Trexler, G. Répássy, and L. Patthy, "A novel COCH mutation, V104del, impairs folding of the LCCL domain of cochlin and causes progressive hearing loss," Journal of Medical Genetics, vol. 41, no. 1, article no. e9, 2004.

[12] M. Kamarinos, J. McGill, M. Lynch, and H. Dahl, "Identification of a novel COCH mutation, I109N, highlights the similar clinical features observed in DFNA9 families," Human Mutation, vol. 17, no. 4, p. 351, 2001.

[13] R. J. Pauw, P. L. M. Huygen, R. W. J. Collin et al., "Phenotype description of a novel DFNA9/COCH mutation, I109T," Annals of Otology, Rhinology and Laryngology, vol. 116, no. 5, pp. 349$357,2007$.

[14] J. Gao, J. Xue, L. Chen, X. Ke, Y. Qi, and Y. Liu, "Whole exome sequencing identifies a novel DFNA9 mutation, C162Y," Clinical Genetics, vol. 83, no. 5, pp. 477-481, 2013.

[15] V. A. Street, J. C. Kallman, N. G. Robertson, S. F. Kuo, C. C. Morton, and J. O. Phillips, "A novel DFNA9 mutation in the vWFA2 domain of $\mathrm{COCH}$ alters a conserved cysteine residue and intrachain disulfide bond formation resulting in progressive hearing loss and site-specific vestibular and central oculomotor dysfunction," American Journal of Medical Genetics A, vol. 139, no. 2, pp. 86-95, 2005.

[16] A. M. L. C. Bischoff, P. L. M. Huygen, M. H. Kemperman et al., "Vestibular deterioration precedes hearing deterioration in the P51S COCH mutation (DFNA9): an analysis in 74 mutation carriers," Otology \& Neurotology, vol. 26, no. 5, pp. 918-925, 2005.

[17] X. Gu, L. Guo, H. Ji et al., "Genetic testing for sporadic hearing loss using targeted massively parallel sequencing identifies 10 novel mutations," Clinical Genetics, vol. 87, no. 6, pp. 588-593, 2015.

[18] R. Grabski, T. Szul, T. Sasaki et al., "Mutations in $\mathrm{COCH}$ that result in non-syndromic autosomal dominant deafness (DFNA9) affect matrix deposition of cochlin," Human Genetics, vol. 113, no. 5, pp. 406-416, 2003.

[19] J. Yao, B. F. Py, H. Zhu, J. Bao, and J. Yuan, "Role of protein misfolding in DFNA9 hearing loss," The Journal of Biological Chemistry, vol. 285, no. 20, pp. 14909-14919, 2010. 

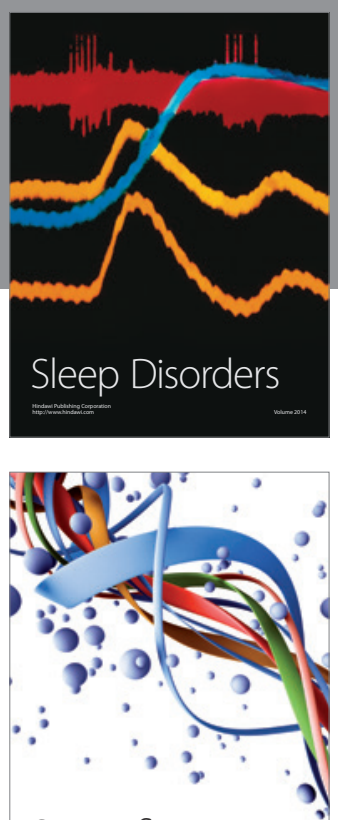

Scientifica
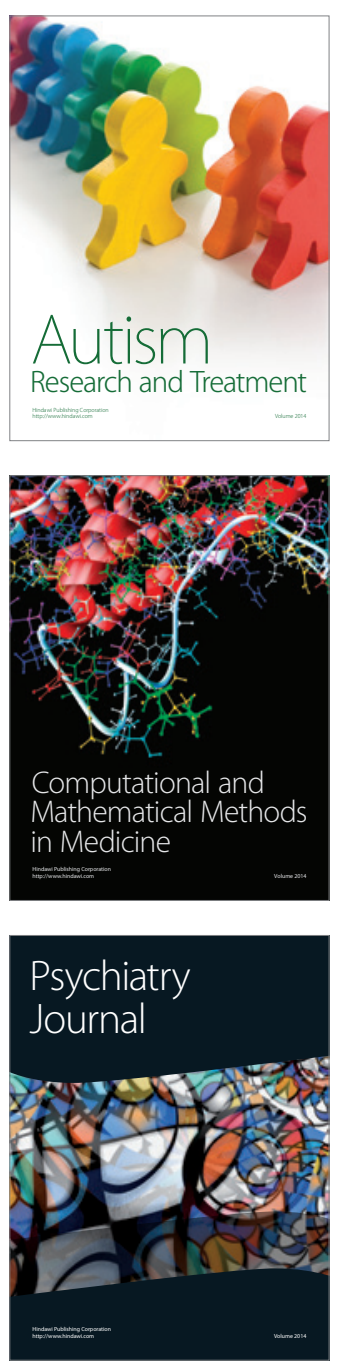
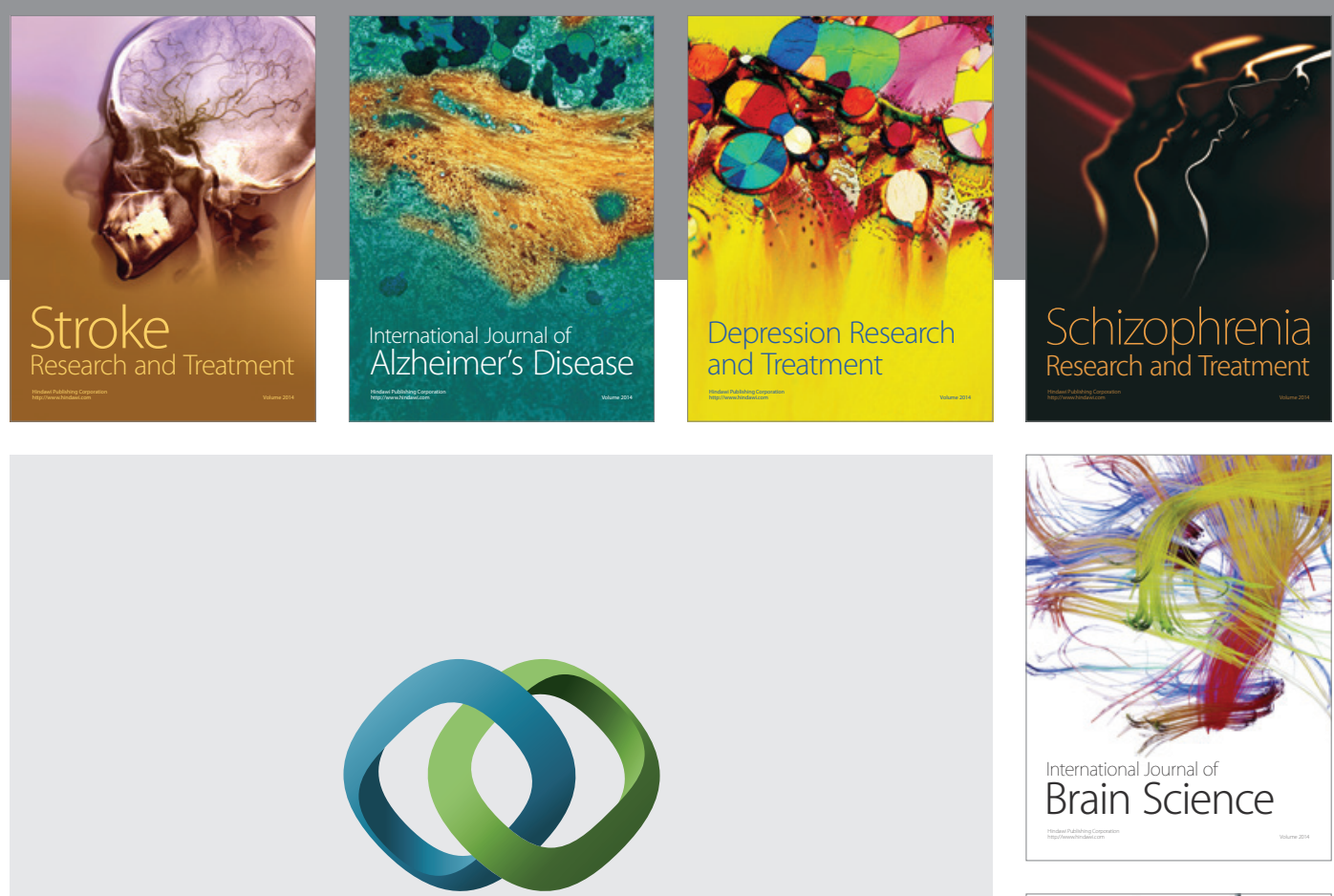

\section{Hindawi}

Submit your manuscripts at

http://www.hindawi.com
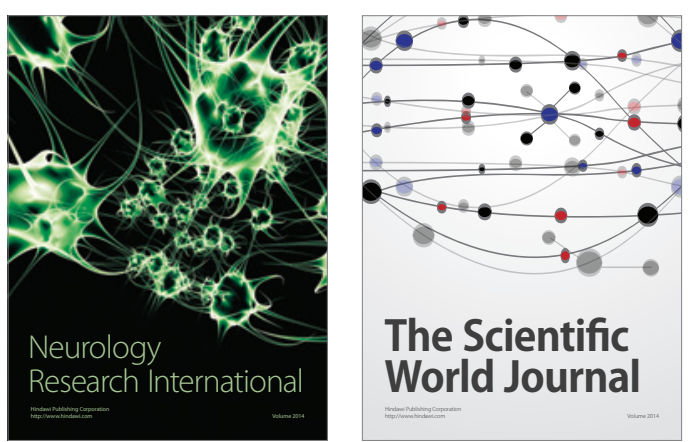

The Scientific World Journal

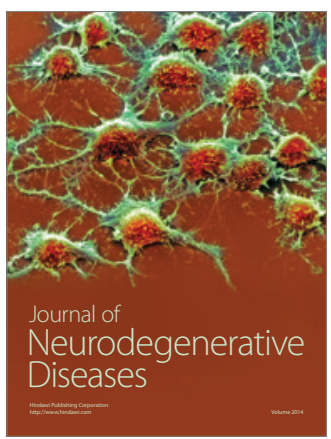

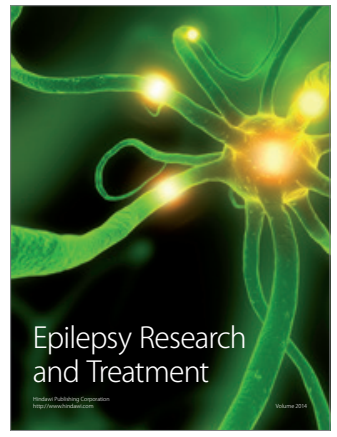

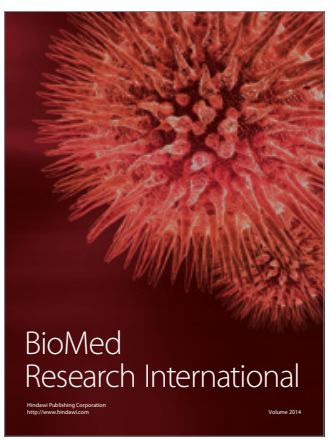

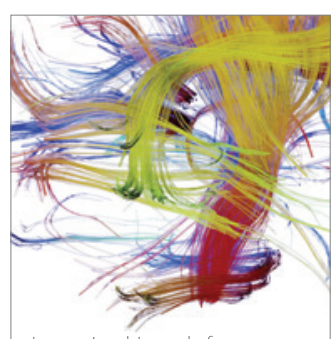

Brain Science

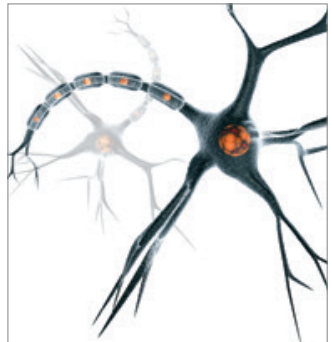

Neural Plasticity
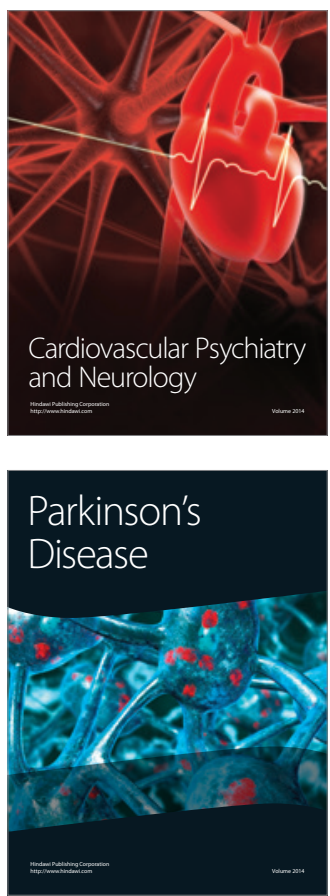